\title{
Thrombophlebitis causing Pyrexia of unknown origin diagnosed by PET-CT
}

\author{
Kamaledeen $\mathrm{S}^{1 *}$, Vardon $\mathrm{L}^{2}$, Poulton $\mathrm{S}^{3}$, Flowers $\mathrm{D}^{4}$ and Ihezue $\mathrm{C}^{4}$ \\ ${ }^{1}$ Specialist Registrar, Clinical Radiology, Queen Alexandra Hospital, Portsmouth, UK \\ ${ }^{2}$ Specialist Registrar Geriatric Medicine, Queen Alexandra Hospital, Portsmouth, UK \\ ${ }^{3}$ Consultant Geriatrician, Queen Alexandra Hospital, Portsmouth, UK \\ ${ }^{4}$ Consultant Radiologist, Queen Alexandra Hospital, Portsmouth, UK
}

\begin{abstract}
Pyrexia of Unknown Origin is defined as a temperature of more than $38.3^{\circ} \mathrm{c}$ on more than one occasion for at least three weeks, with an unclear diagnosis after one week in hospital. We describe a case of an 82-year-old male with Pyrexia of Unknown Origin in which no diagnosis could be established despite multiple investigations. Positron emission tomography computerized tomography (PET-CT) scan, performed to exclude malignancy, revealed the unexpected finding of an infected thrombus as the source of the pyrexia. Our case demonstrates the pivotal role of PET-CT in diagnosing the cause of Pyrexia of unknown origin. PET-CT is now easily accessible to clinicians and should be considered in the search for an elusive diagnosis of Pyrexia of Unknown Origin.
\end{abstract}

\section{Case presentation}

An 82-year-old male was admitted into our hospital following a mechanical fall in which he sustained a left hip intertrochanteric fracture. He was found to have a temperature of $39.6^{\circ} \mathrm{C}$ on admission and was newly confused. He reported a two-week history of back pain, fevers and rigors prior to his fall. He remained febrile in the postoperative period and was investigated for Pyrexia of Unknown Origin (PUO).

His past medical history included Enterococcus Faecalis infective endocarditis of the aortic valve with subsequent replacement six years ago, possibly secondary to a cystoscopy, and history of controlled seizures.

One month prior to admission he had been suffering with recurrent rigors and sweats, episodes of varying confusion and unsteadiness with falls, which led to the fractured hip for which he was admitted. Blood test by his GP within the month prior to his admission showed raised inflammatory markers with C-reactive protein (CRP) of $119 \mathrm{mg} / \mathrm{l}$.

Several underlying diagnoses of PUO were considered: Community acquired Pneumonia, Infective Endocarditis, Discitis, Cerebral Abscess and Malignancy.

Admission chest $\mathrm{x}$-ray showed no consolidation or effusion. Urine microscopy, urine and blood cultures were negative. Post operative chest $\mathrm{x}$-ray showed small left pleural effusion and empirical antibiotic therapy was commenced for possible community acquired pneumonia. Pleural aspirate was negative.

Non-contrast computed tomography (CT) of the head was performed three days post-operatively for the main purpose of excluding a subdural haematoma given the history of pre-operative fall and persistent confusion post-operatively. This revealed no acute intracranial abnormality.
Pre- and post-contrast CT of the abdomen and pelvis performed to exclude discitis given the patient's history of back pain and spiking temperatures, found no discitis or intra-abdominal collection or source of pyrexia.

However, this revealed a non-occlusive thrombus at the confluence of the superior mesenteric vein with the portal vein and splenic vein with no extension of the thrombus into the superior mesenteric vein (SMV). This was not complicated by bowel ischaemia. A proximal inferior mesenteric vein (IMV) thrombophlebitis was noted and since no nearby macroscopic inflammatory process was found, it was postulated that this could represent translocation of gut bacteria from diverticular sigmoid colon, which also raised the question of whether this was due to immunocompromise. There was no colonic diverticulitis or other intra-abdominal inflammatory process to explain the portal venous thrombosis.

Acid Fast Bacilli (AFB) serology, to exclude Tuberculosis, and HIV serology were negative. A thrombophilia screen, Urinary Bence Jones protein and immunoglobulins to investigate for myeloma were within normal limits.

A pre- and post-contrast CT of the brain was performed given the ongoing pyrexia and confusion, to exclude cerebral abscess. This showed no abnormal enhancement, oedema or space occupying lesion.

${ }^{\star}$ Correspondence to: Salma Kamaledeen, Specialist Registrar, Clinical Radiology Queen Alexandra Hospital, Portsmouth, UK, E-mail: salma.kamaledeen@doctors.org.uk

Key words: pyrexia of unknown origin, fever of unknown origin, positron emission tomography, positron emission tomography computed tomography, PET-CT, thrombophlebitis

Received: May 08, 2018; Accepted: May 22, 2018; Published: May 25, 2018 
Given his prior history of infective endocarditis, trans-thoracic and transoesophageal echocardiography were performed but were negative.

Occult malignancy was considered as an underlying diagnosis, given the incidental finding of venous thrombosis in the IMV and SMV confluence with the portal and splenic veins on CT.

Fluorine-18 fluorodeoxyglucose positron emission tomography (F18-FDG PET) combined with CT (PET/CT) was requested with the intention to rule out malignancy.

F18-FDG PET images were acquired from the skull base to the mid-thighs together with low dose CT for attenuation correction and anatomical co-location. This showed no malignancy but demonstrated high activity at the sites of the thrombus within the confluence of the SMV and portal vein as well as the distal IMV, in keeping with infected thrombi.

The patient was treated with anticoagulation and antibiotics with regular microbiology input. His pyrexia resolved, and he was discharged home to complete a further four-week course of broad-spectrum oral antibiotics and low-molecular weight Heparin with Hematology outpatient input.

Repeat F18-FDG PET- CT images acquired two months later demonstrated complete resolution of the IMV and SMV thrombi (Figures 1 and 2).

\section{Discussion}

Pyrexia of unknown origin poses a diagnostic challenge to the clinician. With no localising signs and no apparent initial cause, it can result in a multitude of investigations, protracted hospital stays and invasive procedures in the search for a diagnosis with the diagnosis remaining elusive in up to $50 \%$ of patients [1].

Defined as a temperature higher than $38.3^{\circ} \mathrm{c}$, lasting three weeks with no clear diagnosis after one week of through investigations, it is often subsequently attributed to infection, malignancy or noninfectious inflammatory conditions. PUO may occur in $16-45 \%$ of infections [2].

Investigation of PUO includes detailed history taking, through clinical examination, full blood count and biochemistry, urinalysis, blood and urine culture, basic imaging studies such as chest radiograph and abdominal ultrasonography.

Second line investigations include contrast CT of the abdomen and pelvic, serum antibodies and more invasive investigations such as tissue biopsy, liver biopsy or explorative laparoscopy/laparatomy, the selection of which is guided by clinical and previous findings [2].

Thrombophlebitis of the portal vein, also known as pylephlebitis, is a rare diagnosis that may be the consequence of intra-abdominal infection such as diverticulitis. In most cases it affects the superior
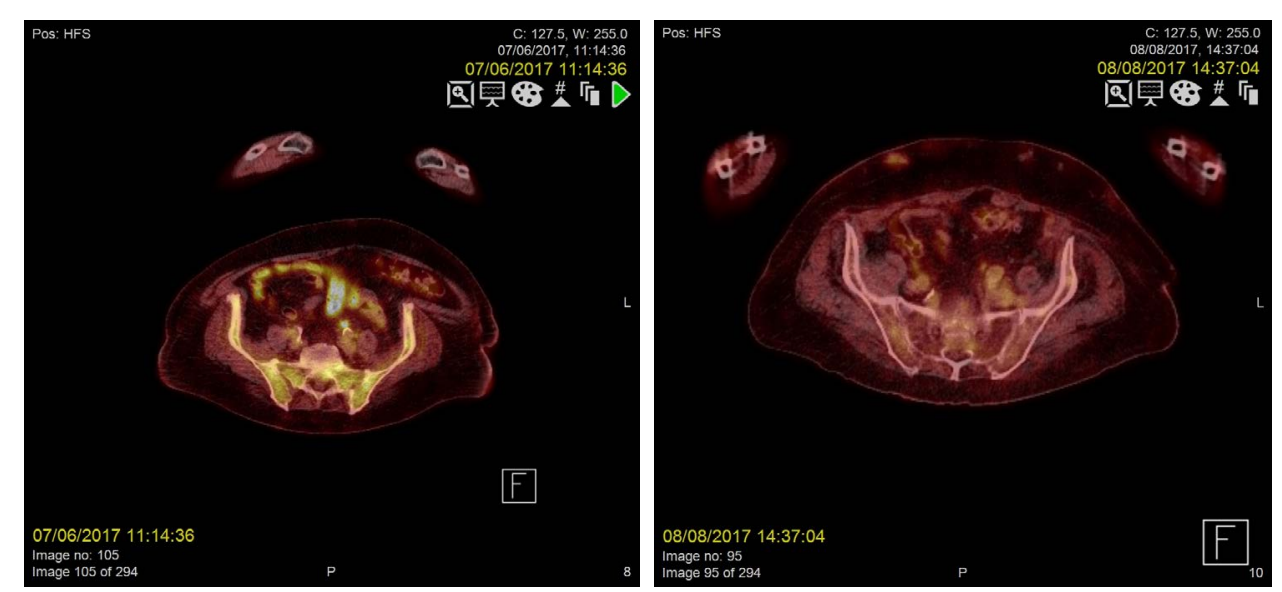

Figure 1. a) Metabolically active, infected thrombus in Inferior mesenteric vein (IMV) demonstrating increased FDG uptake on PET/CT prior to anticoagulant treatment. b) Resolution of infected thrombus in the IMV two months post-treatment
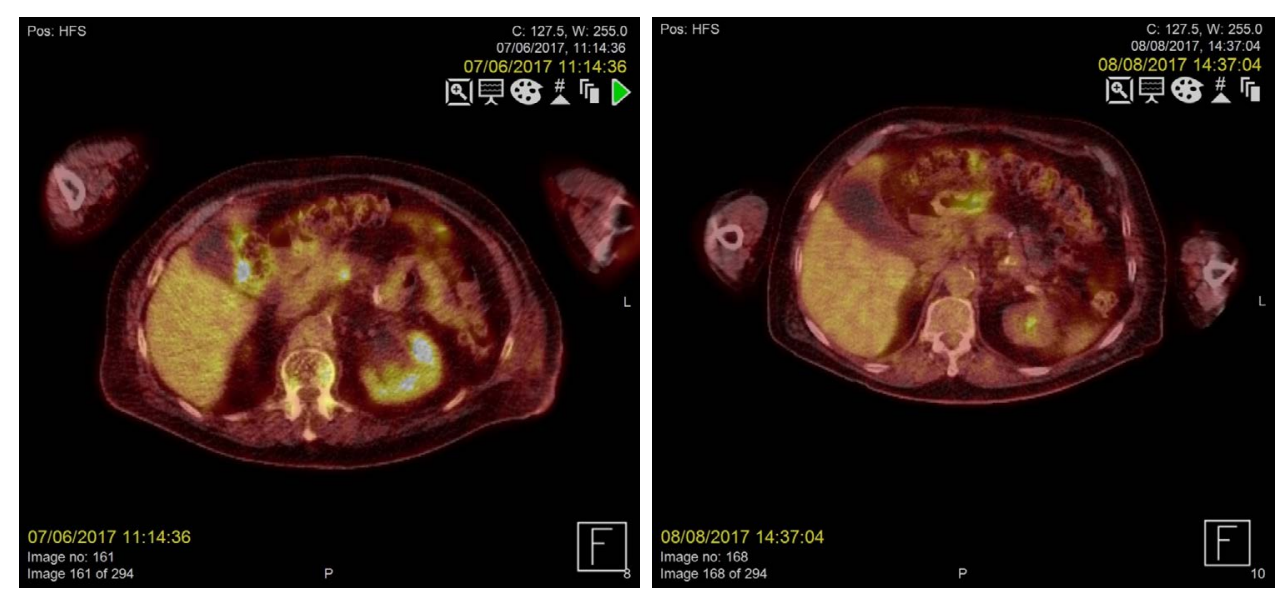

Figure 2. a) Metabolically active, infected thrombus at the confluence of the superior mesenteric vein (SMV) and portal vein demonstrating increased FDG uptake on PET/CT pre-treatment b) Resolution of the infected thrombus at the confluence of the superior mesenteric vein (SMV) on PET/CT two months following treatment 
mesenteric vein $(42 \%)$, closely followed by the portal vein $(39 \%)$ and the splenic vein (12\%) [3].

There is a growing body of literature advocating the use of PET-CT in the initial work-up for PUO. Targeting the three major diagnostic categories for PUO (inflammation, infection or malignancy), it is a superior imaging modality in aiding an accurate and timely diagnosis.

Differentiation between portal or mesenteric vein thrombosis and thrombophlebitis is not possible on CT. A similar case to our own showed that PET scanning led to the diagnosis of portal vein thrombosis after a normal Indium-111-leucocyte scintigraphy with subsequent contrast CT of the abdomen showing thrombosis of the portal vein [4]. Our case demonstrated the diagnosis on combined PET-CT.

CT alone, without PET, yields a diagnosis in PUO in only $19 \%$ of cases [5]. Nuclear medicine studies are useful in detecting disease early before anatomical abnormality becomes apparent. F18- FDG PET combined with CT for co-localisation, is whole body imaging to detect FDG uptake by metabolically active cells in inflammatory, infectious or malignant processes. Although other, more traditional, nuclear medicine studies, eg.radiolabelled leukocyte studies and Gallium 67 scintigraphy may be employed FDG-PET has been shown to have higher sensitivity for identifying the cause of pyrexia of unknown origin than 111In-labelled leuococyte scintography [6] and more sensitive, more specific with faster results than $67-$ Ga scintigraphy $[7,8]$.

Although the specificity of PET-CT is generally low in the range of 23\%-48\% [1], it is more sensitive than FDG PET alone in the diagnosis of PUO $[1,9]$. Overall, FDG PET-CT in patients with PUO has a sensitivity of $85-100 \%$ and detects infections in up to $80 \%$ of patients [2].

Our case demonstrates that FDG PET-CT is invaluable in the diagnosis of complex patients with PUO in whom initial investigations fail to reveal the aetiology. It is for this reason that it is advocated as an early, first line investigation in PUO $[2,10]$.

\section{Conflicts of interest}

None

\section{Funding}

None

\section{Acknowledgement}

Consent was obtained from the patient for publication

\section{References}

1. Kouijzer IJ, Bleeker-Rovers CP, Oyen WJ (2013) FDG-PET in fever of unknown origin. Semin Nucl Med 43: 333-339. [Crossref]

2. Sioka C, Assimakopoulos A, Fotopoulos A (2015) The diagnostic role of $18 \mathrm{~F}$ fluorodeoxyglucose positron emission tomopgraphy in patients with fever of unknown origin. Eur J Clin Invest 45: 601-608.

3. Kanellopoulou T, Alexopoulou A, Theodossiades G, Koskinas J, Archimandritis AJ (2010) Pylephlebitis: an overview of non-cirrhotic cases and factors related to outcome. Scand J Infect Dis 42: 804-811. [Crossref]

4. Bleeker-Rovers CP, Jager G, Tack CJ, Van Der Meer JW, Oyen WJ (2004) F-18fluorodeoxyglucose positron emission tomography leading to a diagnosis of septic thrombophlebitis of the portal vein: description of a case history and review of the literature. J Intern Med 255: 419-423. [Crossref]

5. Quinn MJ, Sheedy PF 2nd, Stephens DH, Hattery RR (1980) Computed tomography of the abdomen in evaluation of patients with fever of unknown origin. Radiology 136 407-411. [Crossref]

6. Seshadri N, Sonoda LI, Lever AM, Balan K (2012) Superiority of 18F-FDG PET compared to 111 In-labelled leucocyte scintigraphy in the evaluation of fever of unknown origin. $J$ Infect 65: 71-79. [Crossref]

7. Meller J, Altenvoerde G, Munzel U, Jauho A, Behe M, et al. (2000) Fever of unknown origin: prospective comparison of [18F] FDG imaging with a double-head coincidence camera and gallium-67 citrate SPET. Eur J Nucl Med 27: 1617-1625. [Crossref]

8. Blockmans D (2004) (18F) Fluoro-deoxyglucose positron emission tomography in patients with fever of unknown origin. Acta Clin Belg 59:134-137.

9. Dibble EH, Yoo DC, Noto RB (2016) Role of PET/CT in Workup of Fever without a Source. Radiographics 36: 1166-1177. [Crossref]

10. Balink H, Verberne HJ, Bennink RJ, van Eck-Smit BL (2012) A Rationale for the Use of F18-FDG PET/CT in Fever and Inflammation of Unknown Origin. Int $J$ Mol Imaging 2012: 165080. [Crossref]

Copyright: (C2018 Kamaledeen S. This is an open-access article distributed under the terms of the Creative Commons Attribution License, which permits unrestricted use, distribution, and reproduction in any medium, provided the original author and source are credited. 